The line $\lambda 3216\left({ }^{3} S_{1}-{ }^{3} P_{0}^{\circ}\right)$ is well resolved in the fifth order, and shows three components decreasing in intensity from the red to the violet and separated by the intervals 0.907 and $0.544 \mathrm{~cm}^{-1}$. Since the ${ }^{3} P_{0}^{\circ}$ term is not split by the nuclear interaction, the triple line-structure corresponds directly to the hyperfine structure of the ${ }^{3} S_{1}$ term. This triplet structure shows that $I \geqslant 1$, and the interval ratio definitely fixes $I$ as $1 \frac{1}{2}$. Theoretically, the interval ratio for a term with $J=1$ is $(I+1) / I$. The observed ratio, obtained by averaging twenty measurements from two plates, is $0 \cdot 907 / 0 \cdot 544=1 \cdot 67$. It is evident that the only value of $I$ that satisfies this ratio is $1 \frac{1}{2}$. The intensities are consistent with this value of $I$ and they, with the intervals, show that the hyperfine levels of ${ }^{3} S_{1}$ are normal.

The lines $\lambda 3190 \quad\left({ }^{3} S_{1}-{ }^{3} P_{1}^{\circ}\right)$ and $\lambda 3109$ $\left({ }^{3} S_{1}-{ }^{3} P_{2}^{\circ}\right)$, although not completely resolved in the fifth order, both show four distinct components. These structures were interpreted by Fisher and Goudsmit's method for incompletely resolved patterns $^{2}$. In both cases the positions and the intensities ${ }^{3}$ of the fine structure components were consistent with the predictions based on the known separations of the ${ }^{3} S_{1}$ term, obtained from $\lambda 3216$, and on the deduced value $1 \frac{1}{2}$ for $I$, which was used to determine the interval ratios of the ${ }^{3} P_{1}^{\circ}$ and ${ }^{3} P_{2}^{\circ}$ terms.

The interval factor of ${ }^{3} S_{1}$ is $A\left({ }^{3} S_{1}\right)=0.363 \mathrm{~cm} .^{-1}$. The interval factors of the other two terms are $A\left({ }^{3} P_{\mathrm{I}}^{\circ}\right)=0.18 \mathrm{~cm} .^{-1}$, and $A\left({ }^{3} P_{2}^{\circ}\right)=0.15 \mathrm{~cm}^{-1}$. The last two were determined by the graphical method and are not so accurate as the first. The approximate equality of $A\left({ }^{3} P_{1}^{\circ}\right)$ and $A\left({ }^{3} P_{2}^{\circ}\right)$ shows that the coupling in the $4 s 5 p$ configuration approximates to the $(L S)$ type ${ }^{4}$. This is consistent with the multiplet separations of the $4 s 5 p^{3,1} P$ terms : although the interval ratio $3 \cdot 2 / 1$ for the ${ }^{3} P_{012}^{\circ}$ separations $^{1}$ differs appreciably from the ratio $2 / 1$, the $g$-factors calculated by Houston's method ${ }^{5}$ depart only slightly from those for $(L S)$ coupling.

The nuclear moment deduced from the hyperfine structures of these As IV lines is in agreement with the value deduced by Tolansky ${ }^{6}$ from the hyperfine structure of As II. His publication appeared during the course of this investigation.

The $4 s 5 p$ configuration of As IV and the $4 p 5 s$ configuration of As II illustrate nicely the influence of binding on coupling. The coupling energy between the $s$ and $p$ electrons is expected to be about the same for both configurations. The (ls) interaction of the $p$ electron in the $4 p 5 s$ configuration is expected to be from two to three times that of the $p$ electron in the $4 s 5 p$ configuration. This follows from an application of the Landé doublet formula when the screening effect of the two $4 s$ electrons ${ }^{7}$ and the variation in $n^{*}$ are taken into consideration. Due to this increase in the $(1 s)$ interaction relative to the interaction energy between the two electrons, the coupling in the $4 p 5 s$ configuration should depart more from $(L S)$ type than the coupling in the $485 p$ configuration. This is consistent with observation: the coupling in $4 s 5 p$, as pointed out, approaches $(L S)$ type, whereas the coupling in $4 p 5 s$ differs considerably from $(L S)$ and tends toward $(j j)$, as shown by the application of Houston's theory ${ }^{5}$ to the multiplet separations and of Goudsmit's theory ${ }^{4}$ to the hyperfine separations of As II.

In this research, hyperfine structure was also observed in several unclassified spark lines. These structures are being investigated.

The authors wish to thank Prof. E. F. Burton, director of the Laboratory, who co-operated and made possible the completion of the investigation by procuring the new grating.

McLennan Laboratory,

M. F. CRAWFORD.

A. M. Crooker.*

University of Toronto. March 10.

* Bursar of the National Research Council of Canada.

K. R. Rao, Proc. Roy. Soc., A, 134, 604; 1931.

R. A. Fisher and S. Goudsmit, Phys. Rev., 37, 1057; 1931

E. L. Hill, Proc. Nat. Acad. Sci., 16, 68; 1930

4 S. Goudsmit, Phys. Rev., 37, 663 ; 1931 .

W. V. Houston, Phys. Rev., 33, $297 ; 1929$.

- S. Tolansky, Proc. Roy. Soc., À, 137, 541; 1932. Nature, 129, $652 ; 1932$.

R.A. Sawyer and C. J. Humphreys, Phys. Rev., 32, 583; 1928.

\section{Photochemical Reaction of Hydrogen and Chlorine}

Prof. BAKker and I have talked over the matter dealt with in his letter to NATURE published in the issue of January 7, p. 27, namely, the influence of traces of water vapour on the above reaction. We agree that no further discussion of the point at issue would be useful unless combined with a simultaneous firsthand and detailed examination of the experimental method used. This is impossible, as the apparatus in question has been dismantled. We therefore, for the time being at all events, hold to our respective and divergent views on the subject.

About three years ago Dr. J. B. Bateman, working in this Laboratory with dispersed monochromatie light, obtained results which indicated that the quantum efficiency for this reaction fell off in the ultra-violet, and became practically zero somewhere below $3000 \mathrm{~A}$. Recent experiments carried out by Mr. H. C. Craggs under rather different conditions have shown that the results were due to the presence of a plate of glass, instead of quartz, at the exit side of the thermopile mounting in which the telescope slit of the monochromator is incorporated. This in turn was due to an unfortunate misunderstanding for which we can take no responsibility. In view of the extraordinary nature of the original conclusion, Dr. Bateman's work was not published in full, pending further confirmation. The results have, however, been quoted on two occasions - at a Faraday Society discussion in April 1931 and in a contribution to a Chemical Society discussion held in December 1931. On that account $I$ think that a formal withdrawal is necessary.

King's College,

Strand, W.C.2.

March 31

\section{Sacred Sandstone of the Mayas}

Axx archæologists are familiar with the work done on the remarkable Maya ruins at Chichen Itzá, in Yucatan, by Mr. Earl Morris and his associates, on behalf of the Carnegie Institution of Washington (see Nature, 128, 692, Oct. 24, 1931). In the course of uncovering the large building now known as the Temple of the Warriors, Mr. Morris found an older temple underneath it, which he was able to explore by means of suitable tunnels. It was in the basement of this older building that he found the beautiful turquoise plaque, now carefully restored so far as the materials permitted, and deposited in the National Museum of Mexico. The centre of this plaque consists of a circular area which 